\title{
The effectiveness of alprostadil in treating coronary microcirculation dysfunction following ST-segment elevation myocardial infarction in a pig model
}

\author{
TIANBING DUAN $^{1 *}$, JINXIA ZHANG $^{1 *}$, RANRAN KONG $^{1}$, \\ RUI SONG ${ }^{1}$, WEILONG HUANG ${ }^{2}$ and DINGCHENG XIANG ${ }^{1}$ \\ Departments of ${ }^{1}$ Cardiology and ${ }^{2}$ Ultrasonography, \\ General Hospital of Southern Theater Command, Guangzhou, Guangdong 510010, P.R. China
}

Received May 7, 2019; Accepted November 19, 2020

DOI: $10.3892 /$ etm.2021.10884

\begin{abstract}
Though alprostadil has been reported to improve the impaired microcirculation of patients with pulmonary arterial hypertension, its effectiveness as a treatment for coronary microvasculature dysfunction (CMD) following ST-segment elevation myocardial infarction (STEMI) is unknown. A total of 18 miniature pigs with CMD following STEMI were randomized into three groups that received an intracoronary injection of $5 \mathrm{ml}$ of normal saline, $2 \mathrm{mg}$ of nicorandil or $10 \mu \mathrm{g}$ of alprostadil immediately after measurement of the index of microcirculatory resistance (IMR) and then an intravenous drip containing $5 \mathrm{ml}$ of normal saline, $2 \mathrm{mg}$ of nicorandil or $10 \mu \mathrm{g}$ of alprostadil once a day for 6 days. The IMR, cardiac function using ultrasound, infarct areas and heparanase levels in infarct areas were measured and compared between the three groups. The IMR decreased markedly 10 min after alprostadil or nicorandil intracoronary injection (both $\mathrm{P}<0.05$ ) but not following saline injection $(\mathrm{P}>0.05)$. After 7 days, the IMR was substantially lower in the alprostadil and nicorandil groups compared with the saline group (both $\mathrm{P}<0.05$ ) and the ejection fraction was considerably higher in the alprostadil and nicorandil groups compared with the saline group (both $\mathrm{P}<0.05)$. Differences in infarct areas and the relative heparanase expression levels among the 3 groups were similar to the differences in the ejection fraction. No significant differences in the above assessment indexes were identified in the alprostadil and nicorandil groups. Alprostadil infusion improved coronary microcirculation function, reduced the
\end{abstract}

Correspondence to: Professor Dingcheng Xiang, Department of Cardiology, General Hospital of Southern Theater Command, 111 Liuhua Road, Guangzhou, Guangdong 510010, P.R. China E-mail: dcxiang@foxmail.com

${ }^{*}$ Contributed equally

Key words: index of microcirculatory resistance, pig, ST-segment elevation myocardial, coronary microvascular dysfunction infarct area and limited left ventricular dilatation in a pig coronary microvasculature dysfunction model following STEMI.

\section{Introduction}

Coronary microvasculature dysfunction (CMD) exists in patients with ST-segment elevation myocardial infarction (STEMI) undergoing percutaneous transluminal coronary angioplasty or stent implantation, with a prevalence of 5-50\% (1). Current trials have revealed that CMD has a strong impact on prognosis and outcomes, including persistent chest pain following primary percutaneous coronary intervention (PPCI) and reinfarction and is associated with a lower rate of ST-segment resolution of $<50-70 \%$, a higher rate of late-onset heart failure and increased mortality (2-5). Therefore, CMD has important clinical significance in investigating an optimal treatment strategy for patients with CMD following STEMI.

Nicorandil, a coronary vasodilator that acts on both macro- and microvascular systems, has been demonstrated to possess cardioprotective properties, including anti-arrhythmic effects, and to facilitate ischemic preconditioning, prevention of reperfusion injury and treatment of the no-reflow/slow-flow phenomenon during coronary interventions for acute myocardial infarction (6-9). Thus, nicorandil has been recognized as one of the most important drugs for the treatment of CMD following STEMI (10).

Alprostadil is a prostacyclin that has been investigated for the treatment of pulmonary arterial hypertension, chronic renal failure and diabetic microangiopathy and may improve impaired microcirculation (11-13), but the effectiveness of alprostadil for CMD following STEMI has not been reported, to the best of the authors' knowledge.

The index of coronary microcirculatory resistance (IMR) is a pressure-temperature sensor guidewire-based measurement performed during cardiac catherization (14). The IMR is a specific quantitative measurement and the standard used to assess coronary microvasculature function; it shows a high predictive capacity for the extent and severity of myocardial infarction in patients with STEMI $(15,16)$.

Due to the difficulty of studying CMD in patients with STEMI immediately following PPCI, the present study, using 
normal saline as the negative control group and nicorandil as the positive control group, investigated the therapeutic effect of alprostadil on coronary microcirculation function via the IMR of the left anterior descending artery (LAD) of pigs.

\section{Materials and methods}

Experimental protocol. The Institutional Animal Care and Use Committee of General Hospital of Southern Theater Command approved the protocol of the present study and accounted for the level of mortality observed during it (ethical approval reference no. K2016012). All studies were performed in accordance with the ARRIVE (animal research reporting in vivo experiments) guidelines on the reporting of animal experiments (17). A total of 22 wuzhishan miniature pigs (21 males and 1 female; weight, $22.4 \pm 2.7 \mathrm{~kg}$; age, $9.6 \pm 0.7$ months) were provided by Guangzhou Feed Research Institute [production license no. SCXK (Guangdong) 2015-0036] and were used for model establishment. The animal experiment center of General Hospital of Southern Theater Command kept the animals in a clean environment at a temperature of $16-29^{\circ} \mathrm{C}$, on a $12 \mathrm{~h}$ light/dark cycle and at a humidity of $40-80 \%$. Each miniature pig was fed in a single house with its own trough and drinking point, feeding was twice per day (each feed was 3\% of body weight) and 11 water was supplied 5 times per day [use license no. SYXK (Guangdong) 2014-0100; health and epidemic prevention detection qualified batch no. 44614000000085; quality qualified no. 00131138]. Eventually, a model of CMD following STEMI was established successfully in 18 pigs, which were then and randomized to three groups of 6 pigs that received an intracoronary (IC) injection of either normal saline, nicorandil or alprostadil immediately following IMR measurement and then an intravenous drip of $5 \mathrm{ml}$ of normal saline, $2 \mathrm{mg}$ of nicorandil or $10 \mu \mathrm{g}$ of alprostadil, dependent on the group, once per day for 6 days. The IMR, cardiac function using ultrasound, infarct areas and heparanase levels in infarct areas were measured and compared between the three groups. Details of the experimental protocol are summarized in Fig. 1.

After establishing the CMD model, 18 pigs survived and the other 4 pigs died from intractable ventricular fibrillation (2 pigs), reperfusion arrhythmia (1 pig) and cardiogenic shock (1 pigs). The pig with cardiogenic shock was euthanized after showing signs of distress.

CMD model establishment. For the 5 days prior to the experiment, the pigs received aspirin $(5 \mathrm{mg} / \mathrm{kg}$, nightly), clopidogrel $(5 \mathrm{mg} / \mathrm{kg}$, daily), perindopril $(0.2 \mathrm{mg} / \mathrm{kg}$, daily) and atorvastatin calcium $(1 \mathrm{mg} / \mathrm{kg}$, nightly). General anesthesia was induced via intramuscular injection of a mixture of ketamine $(10 \mathrm{mg} / \mathrm{kg})$, xylazine $(0.075 \mathrm{ml} / \mathrm{kg}$, a mixture of haloperidol, xylidinothiazole and dihydroetorphine) and midazolam $(0.5 \mathrm{mg} / \mathrm{kg})$ and then maintained with a mixture of $0.9 \%$ sodium chloride $(0.4 \mathrm{ml} / \mathrm{kg})$, ketamine $(5 \mathrm{mg} / \mathrm{kg})$ and propofol $(2 \mathrm{ml} / \mathrm{kg})$ delivered continuously with a medical syringe pump through a marginal ear vein $(8-18 \mathrm{ml}$ per $\mathrm{h})(18,19)$. No pig was intubated and oxygen was supplied at $2 \mathrm{l} / \mathrm{min}$. Penicillin was injected $30 \mathrm{~min}$ before the experiment. Following a local injection of $10 \mathrm{ml}$ of lidocaine in the inguinal area, the right femoral artery was exposed and isolated after skin incision and the separation of subcutaneous tissue, and a 6-French (6-F) guiding catheter sheath (Cordis Corporation) was placed into the artery. A 6-F JR3.5 guiding catheter (Cordis Corporation) was inserted into the left coronary artery through the arterial sheath. The animals were heparinized $(100 \mathrm{U} / \mathrm{kg}$ IC, with another 2,500 units added every hour). Baseline angiography was performed. Following calibration, a 0.014-inch-diameter coronary pressure wire (St. Jude Medical, Inc.) was advanced into the distal LAD. The mean aortic pressure $(\mathrm{Pa})$, mean distal pressure of the LAD (Pd) and mean transit time (Tmn, in $\mathrm{sec}$ ) of a $3 \times 3-\mathrm{ml}$ bolus of room temperature saline injected into the coronary artery were recorded at baseline and at different time points of reperfusion through the 6-F JR3.5 guide catheter and the pressure wire. A maximal hyperemic condition was induced by an IC bolus of papaverine $(18 \mathrm{mg})$ and an IC bolus of nitroglycerine $(200 \mu \mathrm{g})$ was administered before each IMR measurement. After baseline measurements, a balloon angioplasty catheter (Maverick, $2.0 \times 20 \mathrm{~mm}$, Boston Scientific) was advanced to a location between the first and second diagonal branches of the LAD through the 6-F guiding catheter. Coronary blood flow was completely interrupted by balloon inflation and documented via contrast angiography. Balloon deflation was performed under the same protocol in all animals. In all cases, a 12-lead real-time electrocardiogram (ECG) monitoring system (IVT Technology Corporation) recorded the total ECG waveform, including premature ventricular contractions, ventricular tachycardia, ventricular fibrillation and ECG ST and T wave changes.

To reduce the incidence of ventricular tachycardia/ventricular fibrillation following coronary blood flow disruption, ischemic preconditioning was performed continuously, with ischemia for 1, 2, 3 and 5 min before balloon inflation and 1-, 2-,3- and 5-min intervals between occlusion and reperfusion. All animals received $0.15 \mathrm{~g}$ of amiodarone via intravenous (IV) administration, followed by continuous infusions of amiodarone at $60 \mathrm{mg} / \mathrm{h}$ delivered using a medical syringe pump immediately following balloon inflation. The pump was not stopped until balloon deflation and $30 \mathrm{~min}$ of reperfusion. If frequent premature ventricular contractions or brief ventricular tachycardia was observed, then lidocaine $50 \mathrm{mg}$ IV was administered at once. If ventricular tachycardia or fibrillation occurred, then electrical defibrillation at $200 \mathrm{~J}$ was performed at once. If heart failure occurred, then cedilanid $(0.2 \mathrm{mg})$ or furosemide $(20 \mathrm{mg})$ was administered at once. Dopamine (3 $\mathrm{mg})$ was injected to treat low blood pressure. To treat unstable vital signs, epinephrine $(1 \mathrm{mg})$ or atropine $(1 \mathrm{mg})$ were injected.

Interventions during and following the operation. During the procedure, alprostadil was injected IC slowly over $2 \mathrm{~min}$. The other solutions were given as an IC bolus and 10 min later, the IMR of the LAD was measured. After their wounds were sutured and bandaged, the pigs were returned to the animal laboratory. Within 3 days after the operation, 4.8 million units of penicillin was intramuscularly injected. During the next six days, under anesthesia, the pigs received the three indicated solutions once a day at the same dose that they originally received as IC infusions injected into the jugular vein. A 


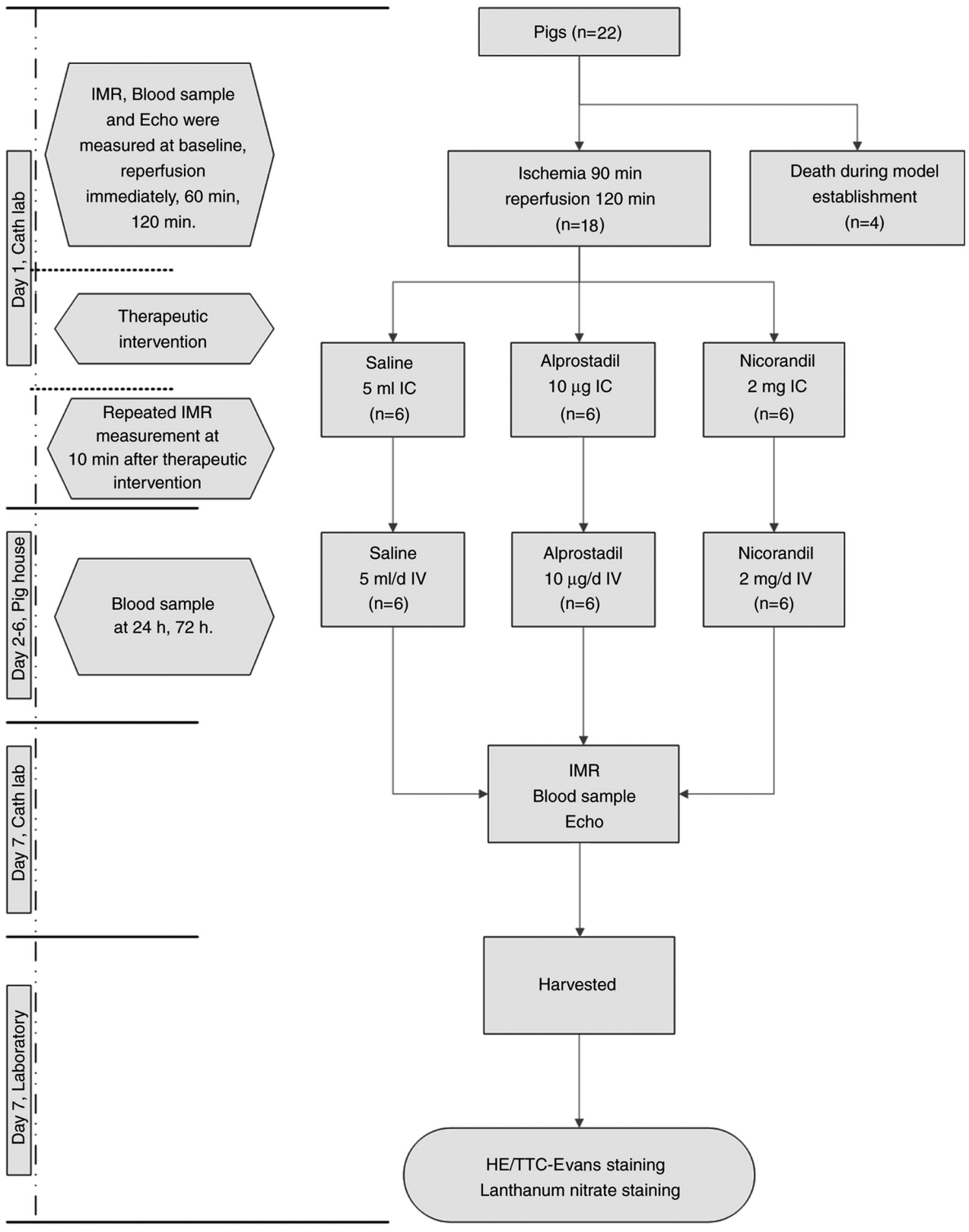

Figure 1. Outline of the therapeutic schedule for studying coronary microcirculation dysfunction in pigs. IMR, index of microcirculatory resistance; Echo, echocardiography; IC, intracoronary; IV, intravenous; Cath lab, catheterization laboratory; HE, haematoxylin and eosin.

6-F JR3.5 guide catheter was placed into the right coronary artery through an arterial sheath to measure the IMR of the LAD on the 7th day.

IMR assessment. The IMR was measured at baseline, balloon occlusion $90 \mathrm{~min}$, after 1,2 $\mathrm{h}$ of reperfusion, $10 \mathrm{~min}$ following the interventions during the operation and on the 7th day after the operation.

Biochemical assessment. Serum levels of cardiac troponin I (cTnI), brain natriuretic peptide (BNP) and endothelin-1 (ET-1) were measured using test kits (cTnI cat. no. N28016833, BNP cat. no. M25016835 and ET-1 cat. no. M25016837, respectively) from the Wuhan Huamei Biological Engineering Research Center. The serum level of nitric oxide (NO) was measured using a test kit (cat. no. 20171018) from the Nanjing
Jiancheng Biological Engineering Research Center. All measurements were performed in duplicate and the values were averaged.

Ultrasound cardiogram. The left ventricular ejection fraction (EF), left ventricular anterior wall thickness (LVAWT), left ventricular end-systolic dimension (LVDs) and left ventricular end-diastolic dimension (LVDd) were measured at baseline, after $2 \mathrm{~h}$ of reperfusion and on the 7th day following the operation (GE Vivid E9, GE Healthcare).

Myocardial tissue sample staining and western blotting. On the 7th day following the operation, the heart was removed, and the infarct area and non-ischemic area of the left ventricle were accurately cut $\left(<1 \mathrm{~cm}^{3}\right)$, then fixed in $10 \%$ formaldehyde fixative solution for $24 \mathrm{~h}$ at $25^{\circ} \mathrm{C}$, dehydrated with ethanol, 
Table I. Baseline characteristics of the three groups.

\begin{tabular}{|c|c|c|c|c|}
\hline Characteristic & SHAM $(n=6)$ & $\operatorname{NCR}(n=6)$ & $\operatorname{ALP}(n=6)$ & P-value \\
\hline Body weight, kg & $22.2(20.0-24.3)$ & $24.6(22.2-27.0)$ & $20.5(17.9-23.3)$ & 0.230 \\
\hline Male, $\%$ & 100 & 83.3 & 100 & 0.358 \\
\hline Age, months & $9.6(9.1-9.9)$ & $9.6(9.2-10.0)$ & $9.6(9.3-9.9)$ & 0.988 \\
\hline Diameter of LAD, mm & $2.1(1.7-2.5)$ & $2.0(1.7-2.2)$ & $2.0(1.8-2.2)$ & 0.654 \\
\hline Ventricular tachycardia or fibrillation, times & $2.5(0.9-4.1)$ & $1.7(0.4-2.9)$ & $1.5(0.4-2.6)$ & 0.370 \\
\hline Electrical Defibrillation, times & $4.0(1.6-6.4)$ & $2.4(0.6-4.4)$ & $3.3(0.5-7.6)$ & 0.668 \\
\hline Baseline $\mathrm{Pa}, \mathrm{mmHg}$ & $114(107-121)$ & $104(94-114)$ & $108(97-121)$ & 0.244 \\
\hline Baseline $\mathrm{Pd}, \mathrm{mmHg}$ & $112(106-118)$ & $102(93-109)$ & $106(95-118)$ & 0.126 \\
\hline Baseline HR, beats/min & $83(74-92)$ & $84(78-91)$ & $77(69-85)$ & 0.211 \\
\hline Baseline IMR & $11.5(9.6-13.3)$ & $12.7(9.4-15.9)$ & $11.4(8.7-14.2)$ & 0.658 \\
\hline Baseline CFR & $4.1(3.6-4.6)$ & $3.9(2.8-4.5)$ & $3.7(3.3-4.1)$ & 0.395 \\
\hline Baseline FFR & $0.94(0.86-0.99)$ & $0.92(0.86-0.97)$ & $0.94(0.92-0.98)$ & 0.571 \\
\hline
\end{tabular}

Values are presented as median (interquartile range, 5th-95th) or rate. $\mathrm{P}<0.05$ indicates a statistically significant difference among the three groups. LAD, left anterior descending coronary artery; Pa, mean aortic pressure; Pd, mean distal pressure of LAD; HR, heart rate; IMR, index of microcirculatory resistance; FFR, fractional flow reserve; CFR, coronary flow reserve; SHAM, pigs treated with saline solution; ALP, pigs treated with alprostadil; NCR, pigs treated with nicorandil.

embedded in paraffin, and paraffin sections were cut into 4- $\mu \mathrm{m}$ slices for H\&E staining (20) (Beijing Solarbio Science \& Technology Co., Ltd.,), TTC-Evans blue staining (20) (Sigma-Aldrich, Merck KGaA) and lanthanum nitrate staining (21) (Electron Microscopy, China), as previously reported.

Heparanase protein levels in infarct areas were detected by western blotting. Myocardial tissues from infarct areas of pig hearts were fully lysed with RIPA Lysis buffer (Thermo Fisher Scientific, Inc.) and the mixture was centrifuged at $300 \mathrm{x} \mathrm{g}$ for $15 \mathrm{~min}$ at $4^{\circ} \mathrm{C}$. The supernatant was collected for western blotting. Protein concentrations were determined using a BCA quantitative test kit (cat. no. BL8890; Bioworld Technology, Inc.). A $30 \mu \mathrm{g}$ protein sample from each group each sample was loaded for $10 \%$ SDS-PAGE electrophoresis and transferred on to nitrocellulose membranes. Membranes were blocked in 5\% BSA solution (cat. no. BS2304; Bioworld Technology, Inc.) for $\geq 60 \mathrm{~min}$ at room temperature and then incubated overnight at $4^{\circ} \mathrm{C}$ with rabbit polyclonal anti-heparanase antibody (1:2,000; cat. no. bs-1541R; BIOSS) and rabbit monolonal anti-GAPDH antibody $(1: 2,000$; cat. no. bs-2188R; BIOSS) with agitation. The next morning, the membranes were washed and incubated with the appropriate horseradish peroxidase-conjugated goat anti-rabbit IgG (1:2,000; cat. no. ab6721; Abcam) for $1 \mathrm{~h}$ at room temperature. The membranes were then washed three times with TBS $-0.05 \%$ Tween-20 and protein bands were visualized using the Western Bright ECL kit (cat. no. K-12045-D50; Advansta). The relative expression of the protein bands was quantified on images obtained with a digital camera (Nikon D7100; Nikon Corporation) using ImageJ (version 6; National Institutes of Health).

Statistical analyses. All statistical analyses were performed using SPSS, version 22.0 (IBM Corp.). Categorical data are presented as a frequency or a percentage and differences among groups were analyzed by $\chi^{2}$ tests. Continuous data are presented as the median with standard deviation. Differences in data measured at different time points were analyzed by repeated-measures ANOVA. If the overall difference was statistically significant, a pairwise comparison was carried out and a Tukey post hoc test was applied. $\mathrm{P}<0.05$ was considered to indicate a statistically significant difference.

\section{Results}

No significant differences were observed among the three groups in the mean body weight, age, diameter of the LAD and baseline $\mathrm{Pa}, \mathrm{Pd}, \mathrm{HR}, \mathrm{IMR}$, coronary flow reserve (CFR) and fractional flow reserve (all $\mathrm{P}>0.05$ ). The baseline characteristics of the three groups of animals are presented in Table I and detailed information is provided in Table SI.

No significant difference in the IMR was observed after $2 \mathrm{~h}$ of reperfusion among the three groups $(\mathrm{P}>0.05)$. Following alprostadil or nicorandil injection, the IMR decreased markedly 10 min later (both $\mathrm{P}<0.05$ ), but not after normal saline injections $(\mathrm{P}>0.05)$. After 7 days, the IMRs in the alprostadil and nicorandil groups were notably lower compared with the normal saline group (both $\mathrm{P}<0.05$ ). Moreover, no difference in the IMR was observed between the alprostadil and nicorandil groups. Furthermore, an equivalence test was performed between the IMRs of the two experimental groups and only one IMR value was beyond the $95 \%$ conformance scope, with a mean difference of 1.63 and a standard deviation of 3.47 (Fig. 2).

Throughout the course of myocardial infarction, the serum concentration of cTnI was lowest at baseline, peaked at $24 \mathrm{~h}$ and dropped sharply at 7 days in all three groups. At 7 days, no significant difference in the serum cTnI was observed among the three groups $(\mathrm{P}>0.05)$. The lowest concentration 

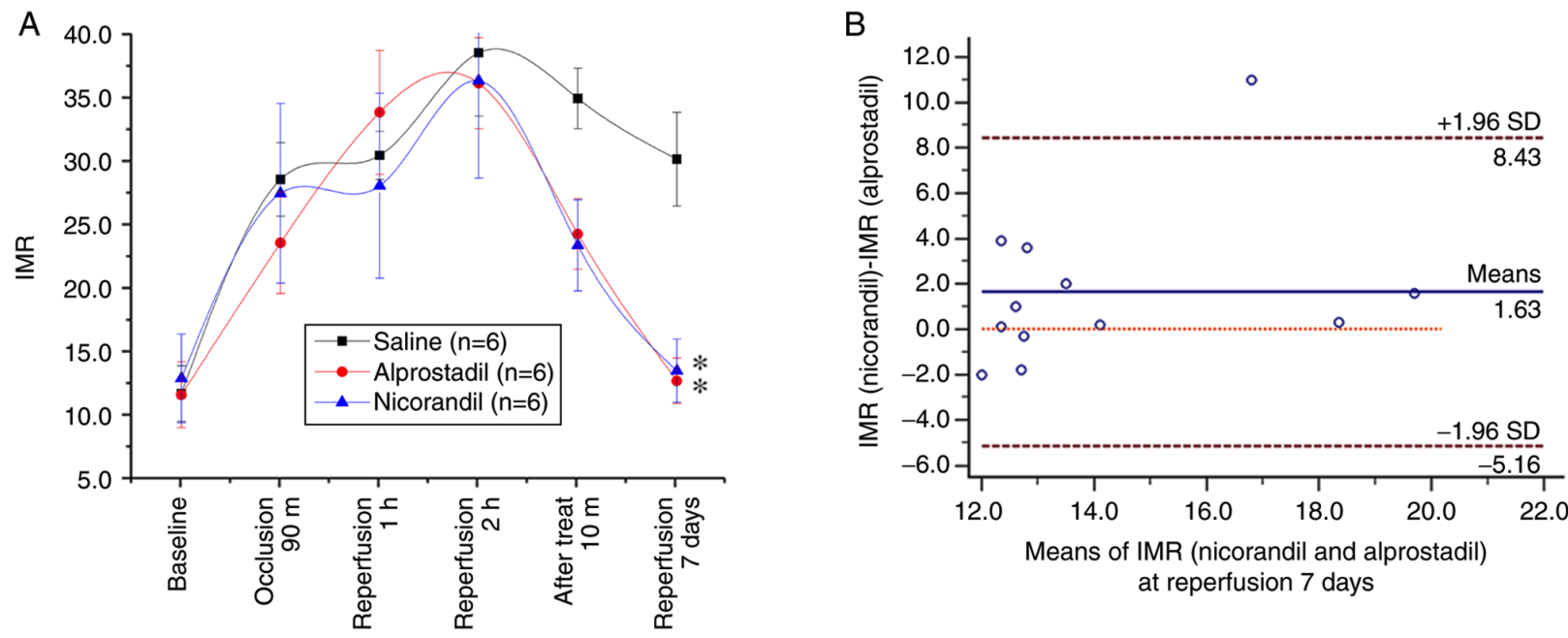

Figure 2. (A) Comparison of the IMR at different time points in the three groups and (B) Bland-Altman plots of the IMR induced by nicorandil and alprostadil.

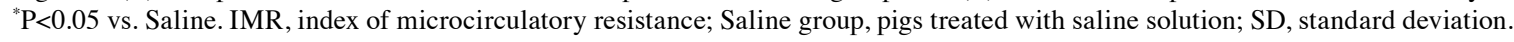
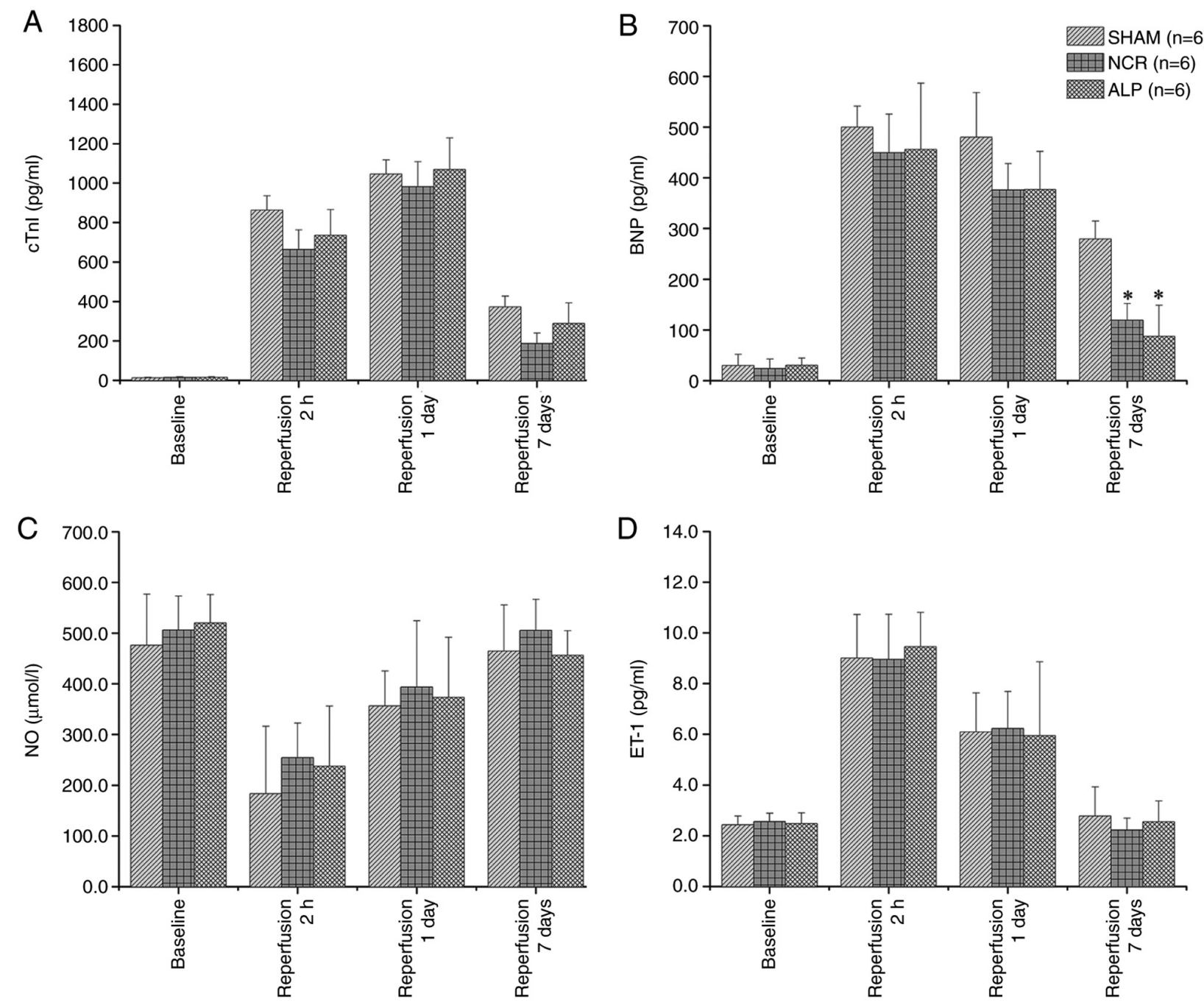

Figure 3. Comparison of the serum concentrations of (A) cTnI, (B) BNP, (C) NO and (D) ET-1 at different time points in the three groups. "P<0.05 vs. SHAM. cTnI, cardiac troponin I; BNP, brain natriuretic peptide; NO, nitric oxide; ET-1, endothelin-1; SHAM, pigs treated with saline solution; NCR, nicorandil; ALP, alprostadil.

of serum BNP was identified at baseline, while the highest level was observed after $2 \mathrm{~h}$ of reperfusion. Compared with the concentration in the normal saline group, a significant difference in the serum BNP concentration was noted in the 

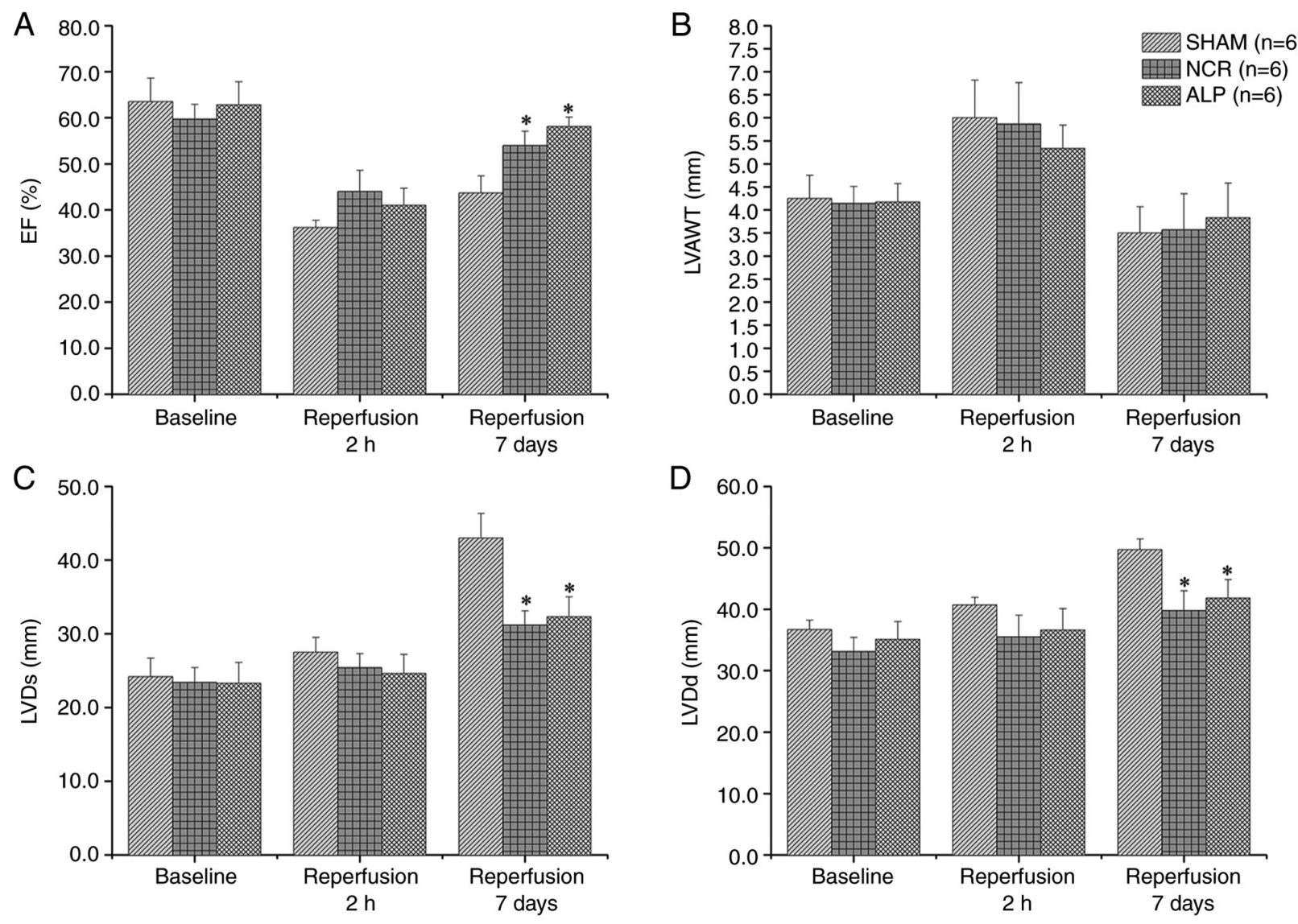

Figure 4. Comparison of the (A) EF, (B) LVAWT, (C) LVDs and (D) LVDd at different time points in the three groups. "P<0.05 vs. SHAM. EF, left ventricular ejection fraction; LVAWT, left ventricular anterior wall thickness; LVDs, left ventricular end-systolic dimension; LVDd, left ventricular end-diastolic dimension; SHAM, pigs treated with saline solution; NCR, nicorandil; ALP, alprostadil.

alprostadil and nicorandil groups (both $\mathrm{P}<0.05$ ). The serum $\mathrm{NO}$ concentration decreased gradually from baseline to the lowest value at $2 \mathrm{~h}$ after reperfusion and had nearly recovered to the baseline level at 7 days. Similar to cTnI, no significant difference in the serum NO or ET-1 concentration was identified among the three groups (Fig. 3).

No significant difference was identified among the three groups for the EF at baseline or after $2 \mathrm{~h}$ of reperfusion ( $\mathrm{P}>0.05$ for both time points). However, after 7 days, the EF in the alprostadil and nicorandil groups was substantially higher compared with the normal saline group (both $\mathrm{P}<0.05$ ). Regional anterior wall motion abnormalities were observed in the left ventricle after $2 \mathrm{~h}$ of reperfusion in all three groups and the LVAWT was increased. After 7 days, anterior wall edema was reduced and the LVAWT decreased. The differences in the LVDd and LVDs among all three groups were similar to those for the EF (Fig. 4).

Post-mortem analyses confirmed the presence of pathological infarction by H\&E staining. The TTC-Evans blue staining results showed that the infarct areas in the alprostadil and nicorandil groups were evidently smaller compared with those in the normal saline group (both $\mathrm{P}<0.05$; Fig. 5). After 7 days, the vascular endothelial glycocalyx was observed to be degraded and reduced or had even disappeared in some cases. Myocyte necrosis and heparanase protein levels in the alprostadil group and nicorandil groups were much lower than those in the normal saline group ( $\mathrm{P}<0.05$; Fig. 6).

\section{Discussion}

The goal of timely reperfusion therapy with fibrinolytic drugs or PPCI in patients with STEMI is to restore blood flow to ischemic areas and reduce mortality (22). With improvements in PPCI technology and progress in the standardized treatment process for STEMI, a considerably higher proportion of patients can achieve successful restoration of epicardial coronary artery patency following prolonged occlusion. However, many of these patients do not experience adequate reperfusion at the microvascular level, resulting in high rates of in-hospital mortality and heart failure (23). Therefore, treatment strategies for STEMI should shift from opening the epicardial artery only downstream of the occlusion to opening the microcirculatory system. The phenomenon of CMD is observed not only in animal models but also in clinical practice $(24,25)$. In clinical practice, the mechanism of CMD is not fully understood; possible mechanisms include existing microvasculature dysfunction, ischemic injury, reperfusion injury, distal atherothrombotic embolization and injury-susceptible coronary microcirculation $(26,27)$. As a result, many treatment strategies to prevent or overcome the above mechanisms have been adopted to reduce the occurrence of CMD or to improve the prognosis of patients with STEMI. Several drugs and methods have been reported to be effective for the prevention or treatment of CMD patients and to reduce the incidence of major adverse clinical events, such as metoprolol, 

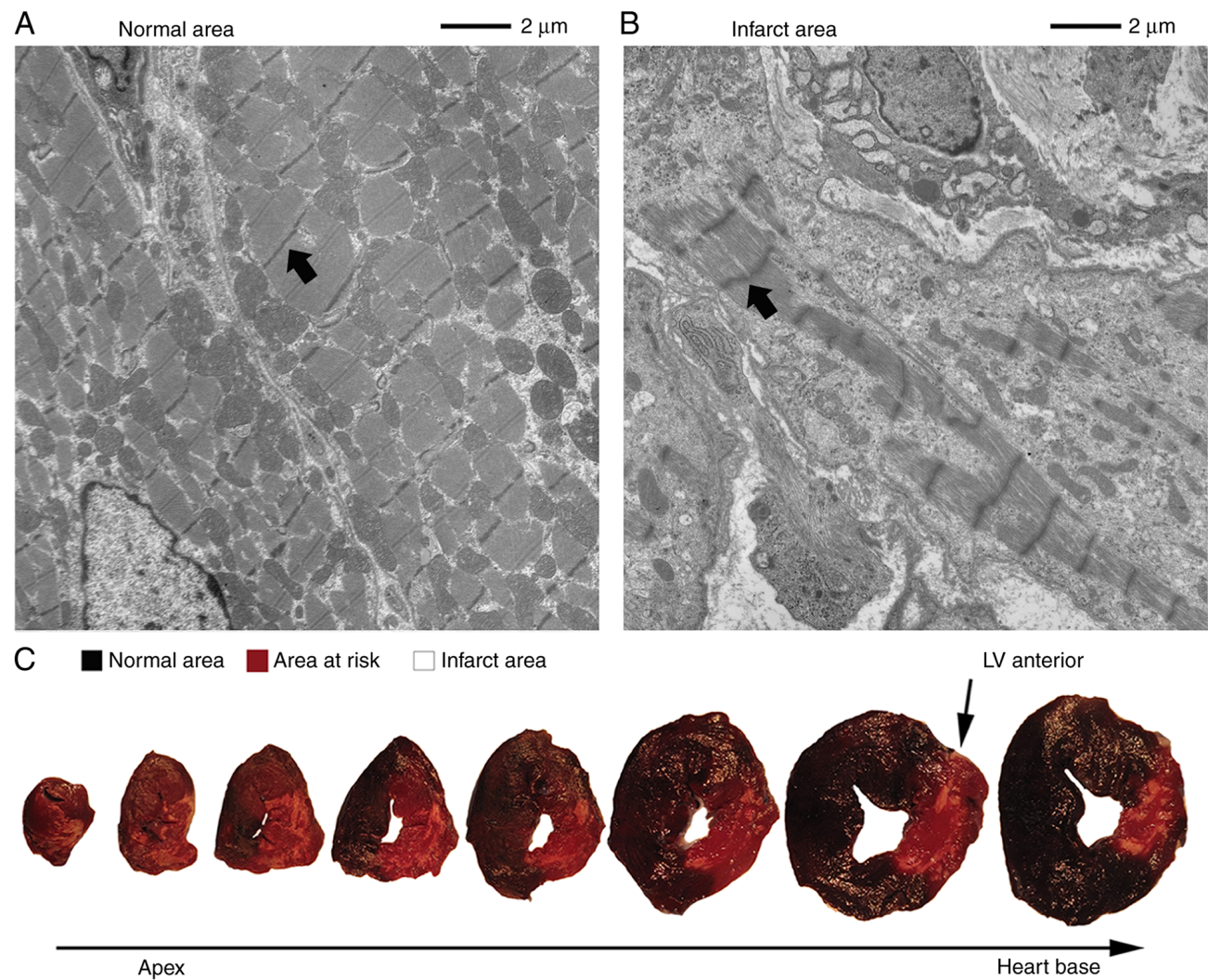

LV anterior

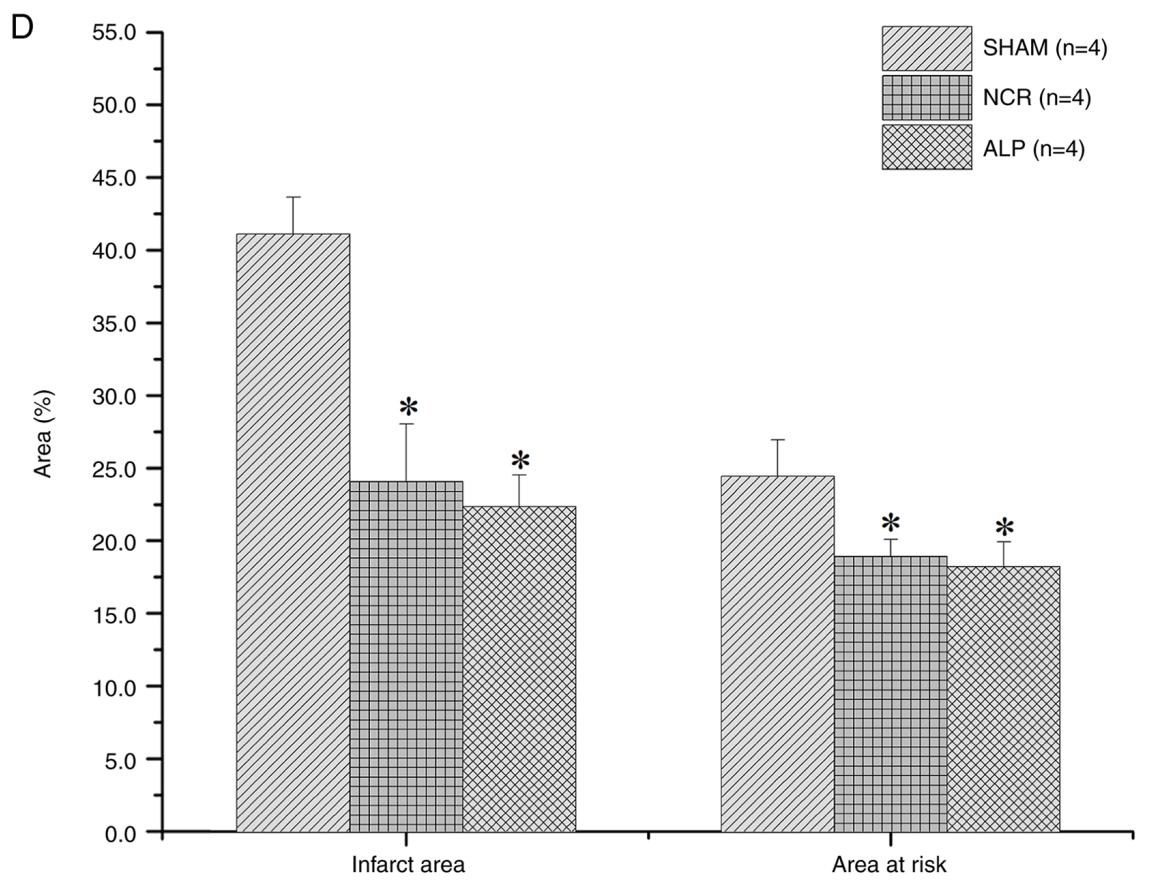

Figure 5. Variation in (A) normal myocardial tissue and (B) infarction tissue 7 days after surgery via lanthanum nitrate staining under an electron microscope and $(\mathrm{C}$ and $\mathrm{D})$ differentiation of infarct areas and areas at risk in the three groups by TTC-Evans blue staining. "P<0.05 vs. SHAM. SHAM, pigs treated with saline solution; NCR, nicorandil; ALP, alprostadil; Black arrow, Z line of sarcomere.

adenosine, nitroprusside, verapamil, nicorandil and deferred stenting (22,28-34).

Nicorandil has a relaxing effect on coronary arteries of different diameters, especially small coronary arteries, and has little influence on blood pressure and heart rate (35). These effects result from the nitrate moiety effect and the ability of nicorandil to open ATP-sensitive potassium channels in coronary arterioles with high resistance, which is associated with increased cellular potassium efflux and decreased extracellular influx (36). In a study testing $12 \mathrm{mg}$ of nicorandil injected into the culprit vessel of patients with STEMI immediately following PPCI, Kostic et al (34) reported that IC nicorandil significantly decreases the IMR and improves the CFR and left ventricular EF in these patients. In a study of 368 patients with STEMI randomly assigned to receive $12 \mathrm{mg}$ of nicorandil or a placebo intravenously immediately before PPCI, Ishii (37) reported that 
A
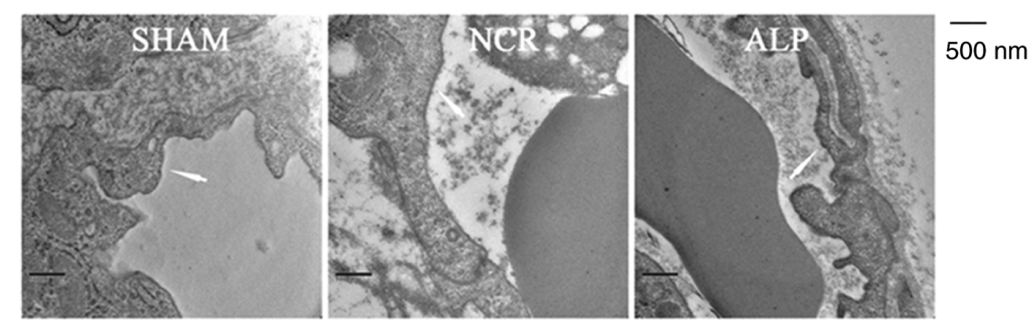

B

SHAM NCR ALP

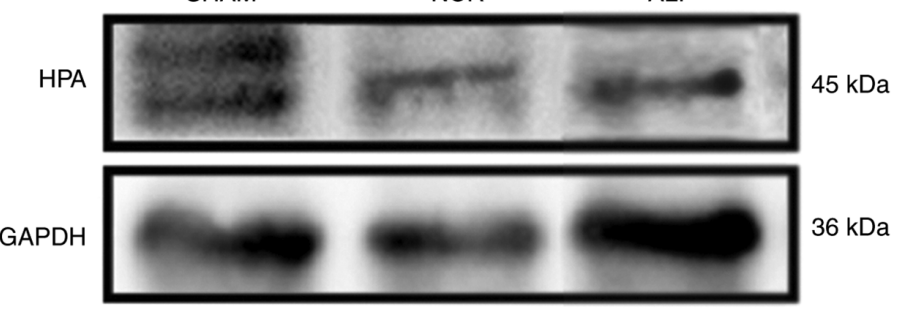

C

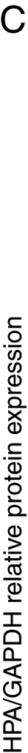

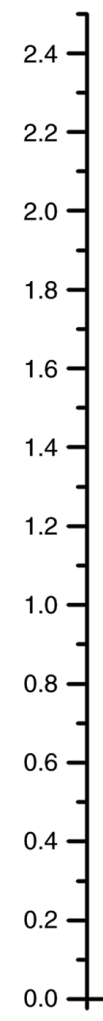

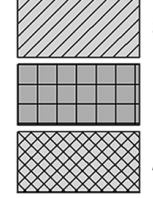

SHAM $(n=3)$

$\operatorname{NCR}(n=3)$ $\operatorname{ALP}(n=3)$

Figure 6. Variations in (A) the glycocalyx of endothelial cells (white arrows) and (B and C) HPA protein levels in infarct areas in the three groups. "P $<0.05$ vs. SHAM. SHAM, pigs treated with saline solution; NCR, nicorandil; ALP, alprostadil; HPA, heparinase.

the rate of ST-segment resolution $>50 \%$ accompanied by lower corrected TIMI frame counts was higher in a nicorandil group compared with the placebo group. After a mean follow-up of 2.4 years, patients treated with nicorandil had a lower incidence of cardiovascular death or rehospitalization for congestive heart failure following PCI (37). Therefore, nicorandil has cardiovascular protective effects and improves coronary microcirculation function. Based on the above evidence, nicorandil was employed as a positive control in this study.

Alprostadil, as a coronary vasodilator that acts on the microvascular system, is reported to be safe and useful in patients with pulmonary hypertension or chronic heart failure and diabetic microangiopathy (11-13). A previous study reported that alprostadil can improve myocardial perfusion in dogs with STEMI. Using a conditioned open-chest dog infarction model, Feld et al (38) reported the effect of alprostadil on coronary blood flow. Alprostadil administered intravenously following copper coil-induced coronary thrombosis in the dog model results in significant acceleration of the thrombolysis time, a significant improvement in coronary blood flow during reperfusion and reduction of the infarct area. After four weeks of IV alprostadil infusion, myocardial perfusion in patients with ischemic heart disease is improved significantly as assessed by positron emission tomography (PET) (39).

The following is an explanation for the dose selection of the three drugs used in the three groups in the present study. 
The animals in the nicorandil group were given $2 \mathrm{mg}$ of nicorandil via IC injection followed by a $2-\mathrm{mg}$ IV injection based on a previous study (8). That is, $2 \mathrm{mg}$ of nicorandil was injected before coronary angiography and $2 \mathrm{mg}$ of nicorandil was injected following stent implantation. Some studies have reported high similarities between pigs and humans for the coronary arterial system with respect to morphology and size and even capillary diameter $(40,41)$. The heart weight of the miniature pigs used in the present study was $\sim 100 \mathrm{~g}$, which is $40-50 \%$ of the heart weight of an adult human; therefore, $2 \mathrm{mg}$ of nicorandil was selected as the positive control. Nicorandil (2 mg) was diluted with normal saline to $5 \mathrm{ml}$ for the IC injection before the IV injection. Therefore, $5 \mathrm{ml}$ of normal saline IC was used for the negative control group. No study has reported IC injection of alprostadil in animal experiments or clinical research, to the best of the authors' knowledge. Hülsmann et al (11) reported that patients with dilated cardiomyopathy and end-stage right ventricular function may be able to tolerate different doses of alprostadil and the continuous IV injection doses were 2.5, 5, 10, 15, 20, 25, 30,35 and $40 \mathrm{ng} / \mathrm{kg} / \mathrm{min}$ until side effects presented, such as headache, nausea and hypotension. Therefore, in the animal experiment, a large IC injection dose was selected and $10 \mu \mathrm{g}$ of alprostadil diluted in $5 \mathrm{ml}$ of normal saline. The 5 - $\mathrm{ml}$ mixture was injected over $2 \mathrm{~min}$. Meanwhile, the heart rate and blood pressure fluctuations in the miniature pigs was closely monitored.

The present study demonstrated the effect of ischemia and reperfusion on coronary microvasculature function in a closed-chest animal model of pigs with STEMI induced by balloon occlusion of the LAD. Coronary microvasculature function before and following normal saline, nicorandil or alprostadil injections was measured using the gold standard angiographic and IMR criteria for the diagnosis of CMD in clinical practice. The main findings of this study can be summarized as follows: i) In an animal CMD model with STEMI, the IMR decreased $10 \mathrm{~min}$ after IC infusions of alprostadil or nicorandil; ii) After 7 days, compared with the negative control group, the IMRs were lower in the alprostadil and nicorandil groups, which also showed a higher EF, smaller LVDs and LVDd values, lower heparanase protein levels and smaller infarct areas. Meanwhile, no significant difference was observed between the nicorandil group and the alprostadil group; and iii) Alprostadil can improve coronary microcirculation function, reduce the infarct area and limit left ventricular dilatation in a pig model of CMD following STEMI.

The IMR is a reliable measurement to assess coronary microvascular dysfunction and changes in the IMR reflect fluctuations of the microcirculation at the different time points in the same vessel (42). Therefore, the changes in the IMR in the present study suggested that IC administration of alprostadil can effectively improve microcirculation conditions, including immediate and short-term effects. The IMR of the miniature pigs in the alprostadil group after $1 \mathrm{~h}$ of reperfusion was higher compared with the normal saline group and nicorandil group, but the difference between the three groups was not statistically significant. It was hypothesized that two reasons may account for this phenomenon. First, a balloon angioplasty catheter was advanced to a location between the first and second diagonal branches of the LAD through a 6-F guiding catheter for model establishment. Coronary blood flow was completely interrupted by balloon inflation as documented by contrast angiography. However, differences were noted in the coronary branches and patterns of each miniature pig. The first diagonal branch of the miniature pigs in the alprostadil group was close to the proximal LAD, which resulted in a relatively large range of myocardial infarction and the IMR of the anterior descending branch was higher compared with the other two groups following reperfusion. Second, the average weight of the miniature pigs in the alprostadil group was approximately 1.7-4.1 kg lower compared with the other two groups. Therefore, it was hypothesized that the slight difference in the weights of the miniature pigs may have affected the IMR of the LAD following acute myocardial infarction. After 7 days, no significant difference was observed in the IMR between the nicorandil group and the alprostadil group, indicating that the effect of alprostadil was equal to that of nicorandil. The change in the IMR following alprostadil injection can be attributed to its pharmacological effect. The known effects of alprostadil include inhibition of vasoconstriction and increased vasodilatation through several regulatory mechanisms, such as inhibition of noradrenaline release from nerve terminals, activation of cAMP, reduction of the concentration of ET-1 and regulation of the NO and ET-1 ratio (43). During balloon inflation, myocardial cell necrosis, endothelial cell debris, microthrombi and some markers of endothelial injury caused distal coronary microvascular spasm or microcirculation obstruction. Following alprostadil injection, microvascular spasm was improved and the microcirculation system was inflated. However, the vasodilation and increase in blood flow following alprostadil administration were small and the vasodilating effect appears to be short term (44). Thus, no significant difference was observed in the changes in serum NO, ET-1 and BNP concentrations among the three groups. The IMR is a real-time indicator of the condition of the coronary microcirculation system $(42,45)$ and the IMR was significantly reduced $10 \mathrm{~min}$ following alprostadil administration.

According to previous studies, the main benefit of alprostadil is an endothelial protective effect, including inhibition of the release of thromboxane A2, which changes the ratio of prostacyclin 2 and thromboxane A2, the interaction between leucocytes and platelets and the inhibition of platelet aggregation, although NO bioavailability is increased $(44,46)$. The endothelial protective effect may develop over a period of at least several days. Thus, 7 days following the procedure, compared with the negative control group, a significant difference in the IMR was identified in the alprostadil group and similar results were observed for the EF, LVDs, LVDd, heparanase protein levels and infarct areas.

In addition, 7 days following surgery, the vascular endothelial glycocalyx was identified to be degraded and reduced or had even disappeared in some cases and myocyte necrosis and heparanase protein levels among the three groups also demonstrated varying degrees of change. Heparan sulfate is a major component of the glycocalyx, which is a coating composed of linear proteoglycan compounds that lines the luminal surface of vascular endothelial cells (47). Heparanase serves a key role in regulating the arterial structure, vascular permeability and the vessel barrier system. Heparanase is the only $\beta$-endo-glucuronidase that specifically cleaves heparan 
sulfate in mammals $(48,49)$. When the level of heparanase increases, more heparan sulfate is cleaved and the barrier function of endothelial cells is impaired (50). Furthermore, loss of function in heparan sulfate elongation genes EXT1 and EXT 2 results in improved NO bioavailability and endothelial function (51). Kawamura et al (52) reported that alprostadil suppresses the production of IL-6 and IL- 8 and the change in the balance between pro-and anti-inflammatory cytokines may be one of the most important cytoprotective mechanisms of alprostadil during cardiac surgery. Notably, heparanase activates macrophages, resulting in the marked induction of cytokine expression associated with endothelial function (53). In the present study, heparanase protein levels in the infarct areas of pigs treated with alprostadil or nicorandil were much lower compared with the pigs that received normal saline. Therefore, it is hypothesized that heparanase may serve a vital role in the pathogenesis of CMD following STEMI in pigs.

As it acts on both the macro- and microvascular systems, nicorandil is one of the most useful drugs for improving coronary microcirculation function. In the present study, compared with the positive control group, no significant difference was identified in the IMR 10 min after IC alprostadil infusion and the IMR 7 days later, indicating that alprostadil can improve immediate and short-term myocardial blood flow in pigs. Meanwhile, similar changes in the EF, LVDs, LVDd, heparanase protein levels and infarct areas showed that alprostadil improved the immediate and short-term coronary microcirculation and has protective effects on the myocardium of pigs with STEMI.

Several limitations existed in the present study. First, the number of pigs used to investigate the effects of the interventions on CMD was small, which may bias the results. Second, due to shortages of research funding and equipment, TTC-Evans blue staining was used to assess differences in infarct areas and ischemic areas in pigs receiving various treatments rather than PET, which is the gold standard for assessing infarct areas. Lastly, the effect of only one dose of alprostadil was studied and whether this dose is optimal for treating microcirculation dysfunction and whether the cardiac protective effect of alprostadil is dose-dependent remains to be elucidated. Additional studies are needed to determine the effects of other treatment doses on improving microcirculation function. In addition, the present study did not identify a clear signaling pathway for the cardioprotective effects of alprostadil, thus rendering it less innovative. Further experiments in pigs are impractical to perform at present, but the signaling pathways affected by alprostadil will be investigated in subsequent studies.

In conclusion, alprostadil infusion improved coronary circulation function, reduced infarct areas and limited left ventricular dilatation in a pig model of CMD following STEMI.

\section{Acknowledgements}

Not applicable.

\section{Funding}

The present study was supported by grants from the National Institute of Health fund 'Establishment and improvement of emergency medical treatment systems for acute myocardial infarction' (grant no. 2016YFC1301201) and 'Research on artificial intelligence diagnosis and treatment model and key technology of acute chest pain disease based on big data' (grant no. 202002020036). The funders had no role in study design, data collection and analysis, decision to publish or preparation of the manuscript.

\section{Availability of data and materials}

The datasets used and/or analyzed during the current study are available from the corresponding author on reasonable request.

\section{Authors' contributions}

TD prepared the experiments, acquired the data and drafted the manuscript; JZ measured the IMR at different time points; RS anaesthetized the pigs; RK prepared blood samples during measurements; WH performed cardiac ultrasound testing and collected experimental data; and DX designed the work, edited the article and approved the final version for submission. TD and DX confirm the authenticity of all the raw data. All authors have read and approved the final manuscript.

\section{Ethics approval and consent to participate}

All procedures performed in the present study were conducted in accordance with the ARRIVE (animal research reporting in vivo experiments) guidelines on the reporting of animal experiments. The Institutional Animal Care and Use Committee of General Hospital of Southern Theater Command approved the protocol of the present study.

\section{Patient consent for publication}

Not applicable.

\section{Competing interests}

The authors declare that they have no competing interests.

\section{References}

1. Niccoli G, Burzotta F, Galiuto L and Crea F: Myocardial No-Reflow in humans. J Am Coll Cardiol 54: 281-292, 2009.

2. Fearon W, Shah M, Ng M, Brinton T, Wilson A, Tremmel JA, Schnittger I, Lee DP, Vagelos RH, Fitzgerald PJ, et al: Predictive value of the index of microcirculatory resistance in patients with ST-segment elevation myocardial infarction. J Am Coll Cardiol 51: 560-565, 2008.

3. Ndrepepa G, Tiroch K, Fusaro M, Keta D, Seyfarth M, Byrne RA, Pache J, Alger P, Mehilli J, Schömig A and Kastrati A: 5-year prognostic value of no-reflow phenomenon after percutaneous coronary intervention in patients with acute myocardial infarction. J Am Coll Cardiol 55: 2383-2389, 2010.

4. Hamirani YS, Wong A, Kramer CM and Salerno M: Effect of microvascular obstruction and intramyocardial hemorrhage by CMR on LV remodeling and outcomes after myocardial infarction: A systematic review and meta-analysis. JACC Cardiovasc Imaging 7: 940-952, 2014.

5. Li J, Li X, Wang Q, Hu S, Wang Y, Masoudi FA, Spertus JA, Krumholz HM and Jiang L; China PEACE Collaborative Group: ST-segment elevation myocardial infarction in China from 2001 to 2011 (the China PEACE-Retrospective Acute Myocardial Infarction Study): A retrospective analysis of hospital data. Lancet 385: 441-451, 2015. 
6. Miyazawa A, Ikari Y, Tanabe K, Nakajima H, Aoki J, Iijima R, Nakayama T, Hatori M, Nakazawa G, Tanimoto S, et al: Intracoronary nicorandil prior to reperfusion in acute myocardial infarction. EuroIntervention 2: 211-217, 2006

7. Matsuo H, Watanabe S, Watanabe T, Warita S, Kojima T, Hirose T, Iwama M, Ono K, Takahashi H, Segawa T, et al: Prevention of no-reflow/slow-flow phenomenon during rotational atherectomy-A prospective randomized study comparing intracoronary continuous infusion of verapamil and nicorandil. Am Heart J 154: 994.e1-6, 2007.

8. Lee HC, An SG, Choi JH, Lee TK, Kim J, Kim JH, Chun KJ, Hong TJ, Shin YW and Lee SK: Effect of intra-coronary nicorandil administration prior to reperfusion in acute ST segment elevation myocardial infarction. Circ J 72: 1425-1429, 2008.

9. Kobatake R, Sato T, Fujiwara Y, Sunami H, Yoshioka R, Ikeda T, Saito $\mathrm{H}$ and Ujihira T: Comparison of the effects of nitroprusside versus nicorandil on the slow/no-reflow phenomenon during coronary interventions for acute myocardial infarction. Heart Vessels 26: 379-384, 2011.

10. Galasso G, Schiekofer S, D'Anna C, Gioia GD, Piccolo R, Niglio T, Rosa RD, Strisciuglio T, Cirillo P, Piscione F and Trimarco B: No-reflow phenomenon: Pathophysiology, diagnosis, prevention, and treatment. A review of the current literature and future perspectives. Angiology 65: 180-189, 2014.

11. Hülsmann M, Stefenelli T, Berger R, Sturm B, Parkner A, Zuckermann A, Woloszczuk W and Pacher R: Response of right ventricular function to prostaglandin E1 infusion predicts outcome for severe chronic heart failure patients awaiting urgent transplantation. J Heart Lung Transplant 19: 939-945, 2000 .

12. Friesenecker B, Tsai AG, Dunser MW, Martini J, Hasibeder W and Intaglietta M: Lowered microvascular vessel wall oxygen consumption augments tissue pO2 during PgE1-induced vasodilation. Eur J Appl Physiol 99: 405-414, 2007.

13. Gupta V, Rawat A and Ahsan F: Feasibility study of aerosolized prostaglandin E1 microspheres as a noninvasive therapy for pulmonary arterial hypertension. J Pharm Sci 99: 1774-1789, 2010.

14. Fearon WF, Balsam LB, Farouque HM, Caffarelli AD, Robbins RC, Fitzgerald PJ, Yock PG and Yeung AC: Nove index for invasively assessing the coronary microcirculation. Circulation 107: 3129-3132, 2003.

15. McGeoch R, Watkins S, Berry C, Steedman T, Davie A, Byrne J, Hillis S, Lindsay M, Robb S, Dargie H and Oldroyd K: The index of microcirculatory resistance measured acutely predicts the extent and severity of myocardial infarction in patients with ST-segment elevation myocardial infarction. JACC Cardiovasc Interv 3: 715-722, 2010

16. Cuculi F, De Maria GL, Meier P, Dall'Armellina E, de Caterina AR, Channon KM, Prendergast BD, Choudhury RP, Forfar JC, Kharbanda RK and Banning AP: Impact of microvascular obstruction on the assessment of coronary flow reserve, index of microcirculatory resistance, and fractional flow reserve after ST-segment elevation myocardial infarction. J Am Coll Cardiol 64: 1894-1904, 2014

17. Kilkenny C, Browne WJ, Cuthill IC, Emerson $M$ and Altman DG: Improving bioscience research reporting: The ARRIVE guidelines for reporting animal research. PLoS Biol 8 : e1000412, 2010

18. Lervik A, Raszplewicz J, Ranheim B, Solbak S, Toverud SF and Haga HA: Dexmedetomidine or fentanyl? Cardiovascular stability and analgesia during propofol-ketamine total intravenous anaesthesia in experimental pigs. Vet Anaesth Analg 45: 295-308, 2018

19. Duan T,Zhang J, Xiang D, Song R and Kong R: Effect of combined anesthesia on Wuzhishan miniature pigs in surgery lasting up to 8 hours. Chin J Comp Med 28: 80-85, 2018 (In Chinese).

20. Liu X, Xie W, Liu P, Duan M, Jia Z, Li W and Xu J: Mechanism of the cardioprotection of rhEPO pretreatment on suppressing the inflammatory response in ischemia-reperfusion. Life Sci 78 2255-2264, 2006.

21. Tachi M, Okada H, Matsuhashi N, Takemura G, Suzuki K, Fukuda H, Niwa A, Tanaka T, Mori H, Hara A, et al: Human colorectal cancer infrastructure constructed by the glycocalyx. J Clin Med 8: 1270, 2019

22. Stone GW, Webb J, Cox DA, Brodie BR, Qureshi M, Kalynych A Turco M, Schultheiss HP, Dulas D, Rutherford BD, et al: Distal microcirculatory protection during percutaneous coronary intervention in acute ST-segment elevation myocardial infarction: A randomized controlled trial. JAMA 293: 1063-1072, 2005.
23. Menees DS, Peterson ED, Wang Y, Curtis JP, Messenger JC, Rumsfeld JS and Gurm HS: Door-to-balloon time and mortality among patients undergoing primary PCI. N Engl J Med 369: 901-909, 2013.

24. Kloner RA, Ganote CE and Jennings RB: The 'No-Reflow' phenomenon after temporary coronary occlusion in the dog. J Clin Invest 54: 1496-1508, 1974.

25. Bekkers SC, Yazdani SK, Virmani R and Waltenberger J: Microvascular obstruction: Underlying pathophysiology and clinical diagnosis. J Am Coll Cardiol 55: 1649-1660, 2010.

26. Herrmann J, Kaski JC and Lerman A: Coronary microvascular dysfunction in the clinical setting: From mystery to reality. Eur Heart J 33: 2771-2781b, 2012.

27. Niccoli G, Scalone G, Lerman A and Crea F: Coronary microvascular obstruction in acute myocardial infarction. Eur Heart J 37: 1024-1033, 2016

28. Bestehorn HP, Neumann FJ, Buttner HJ, Betz P, Stürzenhofecker P, von Hodenberg E, Verdun A, Levai L, Monassier JP and Roskamm H: Evaluation of the effect of oral verapamil on clinical outcome and angiographic restenosis after percutaneous coronary intervention: The randomized, double-blind, placebo-controlled, multicenter Verapamil slow-release for prevention of cardiovascular events after angioplasty (VESPA) trial. J Am Coll Cardiol 43: 2160-2165, 2004.

29. Van't Hof AW, Ten Berg J, Heestermans T, Dill T, Funck RC, van Werkum W, Dambrink JH, Suryapranata H, van Houwelingen G, Ottervanger JP, et al: Prehospital initiation of tirofiban in patients with ST-elevation myocardial infarction undergoing primary angioplasty (On-TIME 2): A multicentre, double-blind, randomised controlled trial. Lancet 372: 537-546, 2008.

30. Niccoli G, Rigattieri S, De Vita MR, Valgimigli M, Corvo P, Fabbiocchi F, Romagnoli E, De Caterina AR, La Torre G, Lo Schiavo P, et al: Open-Label, Randomized, Placebo-Controlled evaluation of intracoronary adenosine or nitroprusside after thrombus aspiration during primary percutaneous coronary intervention for the prevention of microvascular obstruction in acute myocardial infarction: The REOPEN-AMI study (Intracoronary Nitroprusside Versus Adenosine in Acute Myocardial Infarction). JACC Cardiovasc Interv 6: 580-589, 2013.

31. Pizarro G, Fernández-Friera L, Fuster V, Fernández-Jiménez R, García-Ruiz JM, García-Álvarez A, Mateos A, Barreiro MV Escalera N, Rodriguez MD, et al: Long-Term benefit of early Pre-Reperfusion metoprolol administration in patients with acute myocardial infarction: Results from the METOCARD-CNIC trial (Effect of Metoprolol in Cardioprotection During an Acute Myocardial Infarction). J Am Coll Cardiol 63: 2356-2362, 2014

32. Hillegass WB, Dean NA, Liao L, Rhinehart RG and Myers PR: Treatment of no-reflow and impaired flow with the nitric oxide donor nitroprusside following percutaneous coronary interventions: Initial human clinical experience. J Am Coll Cardiol 37: 1335-1343, 2001

33. Carrick D, Oldroyd KG, McEntegart M, Haig C, Petrie MC, Eteiba H, Hood S, Owens C, Watkins S, Layland J, et al: A randomized trial of deferred stenting versus immediate stenting to prevent no- or slow-reflow in acute ST-segment elevation myocardial infarction (DEFER-STEMI). J Am Coll Cardiol 63: 2088-2098, 2014

34. Kostic J, Djordjevic-Dikic A, Dobric M, Milasinovic D, Nedeljkovic M, Stojkovic S, Stepanovic J, Tesic M, Trifunovic Z, Zamaklar-Tifunovic D, et al: The effects of nicorandil on microvascular function in patients with ST segment elevation myocardial infarction undergoing primary PCI. Cardiovase Ultrasound 13: 26, 2015 .

35. Jang HJ, Koo BK, Lee HS, Park JB, Kim JH, Seo MK, Yang HM, Park KW, Nam CW, Doh JH and Kim HS: Safety and efficacy of a novel hyperaemic agent, intracoronary nicorandil, for invasive physiological assessments in the cardiac catheterization laboratory. Eur Heart J 34: 2055-2062, 2013.

36. Costa $\mathrm{AD}$ and Garlid KD: Intramitochondrial signaling: Interactions among mitoKATP, PKCepsilon, ROS, and MPT. Am J Physiol Heart Circ Physiol 295: H874-H882, 2008.

37. Ishii $\mathrm{H}$, Ichimiya $\mathrm{S}$, Kanashiro $\mathrm{M}$, Amano $\mathrm{T}$, Imai $\mathrm{K}$, Murohara T and Matsubara T: Impact of a single intravenous administration of nicorandil before reperfusion in patients with ST-Segment-Elevation myocardial infarction. Circulation 112: 1284-1288, 2005 
38. Feld S, Li G, Amirian J, Felli P, Vaughn WK, Accad M, Tolleson TR, Swenson C, Ostro M and Smalling RW: Enhanced thrombolysis, reduced coronary reocclusion and limitation of infarct size with liposomal prostaglandin E1 in a canine thrombolysis model. J Am Coll Cardiol 24: 1382-1390, 1994.

39. Huang CL, Wu YW, Wang SS, Tseng CD, Chiang FT, Hsu KL, Lee CM and Tzen KY: Continuous intravenous infusion of prostaglandin E1 improves myocardial perfusion reserve in patients with ischemic heart disease assessed by positron emission tomography: A pilot study. Ann Nucl Med 25: 462-468, 2011.

40. Hildebrand F, Andruszkow H, Huber-Lang M, Pape H and van Griensven M: Combined hemorrhage/trauma models in pigs-current state and future perspectives. Shock 40: 247-273, 2013.

41. Kumar M, Kasala ER, Bodduluru LN, Dahiya V, Sharma D, Kumar V and Lahkar M: Animal models of myocardial infarction: Mainstay in clinical translation. Regul Toxicol Pharmacol 76: 221-230, 2016.

42. De Maria GL, Cuculi F, Patel N, Dawkins S,Fahrni G, Kassimis G, Choudhury RP, Forfar JC, Prendergast BD, Channon KM, et al: How does coronary stent implantation impact on the status of the microcirculation during primary percutaneous coronary intervention in patients with ST-elevation myocardial infarction? Eur Heart J 36: 3165-3177, 2015.

43. Gensch C, Clever Y, Werner C, Hanhoun M, Bohm M and Laufs U: Regulation of endothelial progenitor cells by prostaglandin E1 via inhibition of apoptosis. J Mol Cell Cardiol 42: 670-677, 2007.

44. Weiss T, Fischer D, Hausmann D and Weiss C: Endothelial function in patients with peripheral vascular disease: Influence of prostaglandin E1. Prostaglandins Leukot Essent Fatty Acids 67: 277-281, 2002

45. Sirol M, Cescau A, Sideris G, Logeart D, Dillinger JG, Mercadier JJ and Henry P: Index of microcirculatory resistance as an early predictive factor of $\mathrm{Lv}$ remodeling after reperfused myocardial infarction. J Am Coll Cardiol 65: A1934, 2015.
46. Wu CC, Wu CI, Wang WY and Wu YC: Low concentrations of resveratrol potentiate the antiplatelet effect of prostaglandins. Planta Med 73: 439-443, 2007.

47. Baker AB, Groothuis A, Jonas M, Ettenson DS, Shazly T, Zcharia E, Vlodavsky I, Seifert P and Edelman ER: Heparanase alters arterial structure, mechanics, and repair following endovascular stenting in mice. Circ Res 104: 380-387, 2009.

48. Li JP and Vlodavsky I: Heparin, heparan sulfate and heparanase in inflammatory reactions. Thromb Haemost 102: 823-828, 2009.

49. Peterson SB and Liu J: Multi-faceted substrate specificity of heparanase. Matrix Biol 32: 223-227, 2013.

50. Baker AB, Gibson WJ, Kolachalama VB, Golomb M, Indolfi L, Spruell C, Zcharia E, Vlodavsky I and Edelman ER: Heparanase regulates thrombosis in vascular injury and Stent-Induced flow disturbance. J Am Coll Cardiol 59: 1551-1560, 2012.

51. Mooij HL, Cabrales P, Bernelot Moens SJ, Xu D, Udayappan SD, Tsai AG, van der Sande MA, de Groot E, Intaglietta M, Kastelein JJ, et al: Loss of function in heparan sulfate elongation genes EXT1 and EXT 2 results in improved nitric oxide bioavailability and endothelial function. J Am Heart Assoc 3: e001274, 2014.

52. Kawamura T, Nara N, Kadosaki M, Inada K and Endo S: Prostaglandin E1 reduces myocardial reperfusion injury by inhibiting proinflammatory cytokines production during cardiac surgery. Crit Care Med 28: 2201-2208, 2000.

53. Vlodavsky I, Blich M, Li JP, Sanderson RD and Ilan N: Involvement of heparanase in atherosclerosis and other vessel wall pathologies. Matrix Biol 32: 241-251, 2013.

This work is licensed under a Creative Commons Attribution-NonCommercial-NoDerivatives 4.0 International (CC BY-NC-ND 4.0) License. 